\title{
Poly(ethylene oxide)-Based Composite Electrolytes Filled with Periodic Mesoporous Silica for Solid State lonics
}

\author{
Yoichi Tominaga, Masahiro Morita, Shigeo Asai and Masao Sumita* \\ Department of Chemistry and Materials Science, Tokyo Institute of Technology, Ookayama, Tokyo 152-8552, Japan \\ *Corresponding author: msumita@o.cc.titech.ac.jp \\ Received April 9, 2005; Accepted April 19, 2005 \\ (c) 2005 The Society of Rubber Industry, Japan
}

\begin{abstract}
Mesoporous silica (MPS) was used for poly(ethylene oxide) (PEO)-based solid polymer electrolytes as novel inorganic filler. For improvement in ionic conductivity in solid state, a room temperature ionic liquid (IL), 1ethyl-3-methylimidazolium tetrafluoroborate, was introduced into periodic nano-tunnels of MPS, and the modified MPS (IL-MPS) was filled with PEO-LiBF 4 electrolyte. Ionic conductivity of neat-MPS-filled composites was approximately 4-fold higher than that of the original electrolyte. On the other hand, the conductivity was more than 11-fold enhanced by addition of IL-MPS, to be more than $10^{-6} \mathrm{~S} / \mathrm{cm}$ at $30^{\circ} \mathrm{C}$ and at least $10 \mathrm{wt} \%$ silica contents. The conductivity increased with increasing IL-MPS contents, to be a maximum value of approximately $3 \times 10^{-6} \mathrm{~S} / \mathrm{cm}$ at $30^{\circ} \mathrm{C}$ and at $40 \mathrm{wt} \%$. Dynamic mechanical measurements for neat- and IL-MPS composites revealed that the addition of fillers improves storage modulus of PEO-based electrolytes at room temperature. The addition of IL-MPS was able to realize the improvement in both ionic conductivity and storage modulus.
\end{abstract}

Keywords Poly(ethylene oxide), Mesoporous silica, Solid state ionics, Polymer electrolyte, Composite, Ionic liquid, Ionic conductivity, Storage modulus.

\section{Introduction}

Solid polymer electrolytes (SPE) such as poly(ethylene oxide) (PEO)-alkali metal salt mixtures ${ }^{1)}$ have much interest as solid-state alternatives for electrochemical device applications, especially for all-polymer lithium batteries ${ }^{2,3}$. For passed three decades, there have been many studies on PEO-based macromolecular design as SPE materials with mainly reduced degree of the crystallinity and enhanced the local chain mobility ${ }^{4-6)}$. Improvement in salt solubility has been achieved by using metal salts containing fluoride anions such as $\mathrm{CF}_{3} \mathrm{SO}_{3}$, $\mathrm{BF}_{4}, \mathrm{PF}_{6}$, and especially $\mathrm{TFSI}^{7}$. Nevertheless, these materials suffer from a relatively low ionic conductivity at room temperature, to be limited to the $10^{-4} \mathrm{~S} / \mathrm{cm}$ in solid state. Gel-type polymer electrolyte that is swelled with polar organic solvent shows good conductivity, however, it has problems on the mechanical property, electrochemical stability, and safety. Moreover, the conduction in these systems is mainly due to the migration of anions, which should be avoided using for electrochemical applications. A novel conduction mechanism that is not due to the segmental motion of polymer chains is needed for the fast migrations in the solid-state matrix.

It is known that SPE-based composites filled with inorganic compounds ${ }^{8-14)}$ are noteworthy materials because of its good mechanical property, reduction in the degree of crystallinity, high electrochemical stability, and improvement in the conductivity. Weston and Steele have first reported the properties of PEO-based composites in $1982^{8)}$, after that Scrosati et al. have developed that the addition of nano-size ceramic particles such as $\gamma$ $\mathrm{LiAlO}_{2}{ }^{9,10)}, \mathrm{Al}_{2} \mathrm{O}_{3}{ }^{11,13,14)}$, and $\mathrm{TiO}_{2}{ }^{11,12)}$ to the SPE can be effective in the solid state. The addition of ceramic fillers gives rise to the remarkable increase in room temperature conductivity, inhibition of polymer re-crystallization, and decrease in glass transition temperature $\left(T_{g}\right)$. In particular, the addition to the SPE is a unique technique to increase in cation transport number without decrease in the conductivity ${ }^{11-14)}$. It is considered that the enhanced cationic conduction might be caused by the active interface between polymer and filler surface, which is based on the Lewis acid-base nature ${ }^{13,14)}$.

Here, we focused on periodic mesoporous molecular sieves $^{15)}$ for SPE as composite derivatives. The mesoporous materials, which can be synthesized with pore 
sizes from approximately 1.5 to $10 \mathrm{~nm}$, have a wide range of potential applications including: shape-selective catalysis and sorption of large organic molecules, chromatographic separations, and uses as hosts to confine guest molecules and atomic arrays ${ }^{16)}$. Since the hexagonally ordered mesoporous silica (MPS) was first discovered by Kuroda et al. (namely FSM-16) ${ }^{17)}$ and Mobil Corporation (namely M41S) ${ }^{18)}$, there have been many studies on novel synthesis method ${ }^{19,20)}$, hybridization with organic compounds ${ }^{21)}$, and aligned thin-films ${ }^{22)}$, because it has high possibility to develop on the materials science. The MPS has extraordinary physicochemical properties such as extremely large surface area, flawless surface condition, and well-regulated porous structure. To realize fast ionic conduction in solid polymers, the use of MPS for SPE is expected to improve the active interface where predominant cationic conduction occurs. High-ordered nanopores in the MPS are fascinating and useful for guest molecules. There have been studies on the PEO electrolytes containing neat-MPS (MCM-41), which was reported by Kao et al. ${ }^{23)}$. Unfortunately, the simple mixture consisting of PEO, salt, and neat-MPS shows merely average conductivity. In this paper, we report ionic conductivity and dynamic mechanical property of composite PEO electrolytes containing MPS. The fillers used in this study are neat and modified MPS which was filled the inside nanopores with room temperature ionic liquid (IL) ${ }^{24)}$. This study first describes the relation between ionic conductivity and storage modulus of both neat- and IL-MPS composite electrolytes.

\section{Experimental}

\section{Sample Preparations}

Mesoporous silica (MPS) was synthesized by simple sol-gel method using nonionic surfactant $\left(\mathrm{EO}_{20} \mathrm{PO}_{70} \mathrm{EO}_{20}\right.$ block copolymer, $M_{w}=5800$, Aldrich) as template molecules for formation of periodic structures ${ }^{19)}$. The synthesis procedure of the MPS was followed by the same process written in a previous report ${ }^{24)}$. The structural image is shown in Figure 1. For internal modification of the neat-MPS, 1-ethyl-3-methylimidazolium tetrafluoroborate $\left(\mathrm{EMIBF}_{4}\right.$, Aldrich) was used without purification. The chemical structure is also shown in Figure 1. Dried neat-MPS $(0.1 \mathrm{~g})$ was directly mixed with $\mathrm{EMIBF}_{4}$ $(1.5 \mathrm{~g})$ at room temperature. The viscous slurry was heated to $60^{\circ} \mathrm{C}$ with stirring for at least $24 \mathrm{~h}$ and then dried in vacuo at $100^{\circ} \mathrm{C}$ for $12 \mathrm{~h}$. The resulting mixture was centrifuged for $15 \mathrm{~min}$. The precipitate on filter was washed three times by acetone and dried at $100^{\circ} \mathrm{C}$ for $24 \mathrm{~h}$
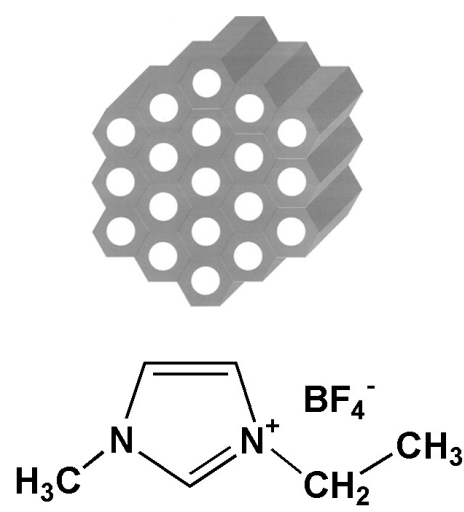

Figure 1. Structural image of a MPS domain (up) and chemical structure of an $\mathrm{EMIBF}_{4}$ molecule (bottom).

(IL-MPS). PEO ( $M_{w}=500000$, Wako Chemical), $\mathrm{LiBF}_{4}$ (Aldrich), and fillers (neat- or IL-MPS) were used for composite films preparation. The starting materials were mixed by the solvent-free method ${ }^{24,25)}$. These were introduced into a polypropylene bottle with small glass balls and it was rolled by ball-milling system for at least $24 \mathrm{~h}$. Resulting homogeneous powder was compressionmolded into an approximately $300 \mu \mathrm{m}$-thick film at $90^{\circ} \mathrm{C}$ for 30 min under dry $\mathrm{N}_{2}$ gas. The PEO- $\mathrm{LiBF}_{4}$ composition of all samples is specified as $5 \mathrm{~mol} \%(\mathrm{OE}: \mathrm{Li}=20: 1)$, which gives the ratio of the number of oxyethylene $(\mathrm{OE})$ units to the number of Li cations. The composite samples with neat-MPS contents from 5 to $20 \mathrm{wt} \%$ and IL-MPS contents from 10 to $40 \mathrm{wt} \%$ (containing the neat-MPS component) have been prepared in this study.

\section{Measurements}

Brunauer-Emmett-Teller (BET) surface area and pore volume of neat-MPS were determined by using a Coulter SA3100 system (Beckman Coulter). Elemental analysis for fillers was carried out using a CHN Corder MT-6 (Yanaco) system. Antipyrine was used for a standard material. Small-angle X-ray scattering (SAXS) measurement for fillers was carried out using a RU-200 system (Rigaku) with $\mathrm{CuK}_{\alpha}$ radiation operated at $50 \mathrm{kV}$ and $180 \mathrm{~mA}$. The SAXS intensity measured by slit-collimation was corrected for parasitic scattering. The scattering angle $(2 \theta)$ range was from 0.2 to 2.0 degree. The sample filler as powder was filled into the center space of a $0.8 \mathrm{~mm}$-thick stainless folder which was sealed with polyethylene thin films (less than $10 \mu \mathrm{m}$-thick) on both sides. Scanning electron microscopy (SEM) photographs were obtained from a FES800 (Hitachi). Sample films were cooled into liquid $\mathrm{N}_{2}$ and fractured. The section was coated with Pt-Pd by an Eiko IB-3 without etching. Ionic conductivity of all 
samples was measured by a complex AC impedance method using a 4192A LF Impedance Analyzer (HewlettPackard) in the frequency range from $100 \mathrm{~Hz}$ to $13 \mathrm{MHz}$. The temperature was increased from 30 to $100^{\circ} \mathrm{C}$ at a heating rate of $2.0^{\circ} \mathrm{C} / \mathrm{min}$ using a temperature controller (KP-1000, CHINO). The sample was sandwiched between two SUS electrodes with a $300 \mu$ m-thick Teflon ${ }^{\circledR}$ spacer, and the cell was then insulated using polyimide tape. The entire process was carried out in a SUS box filled with dry $\mathrm{N}_{2}$ gas. Dynamic mechanical analysis (DMA) was carried out using an IT-DVA 200s (IT Keisoku). The measurement was performed in tension mode with $0.1 \%$ strain amplitude at a frequency of $10 \mathrm{~Hz}$. The temperature was increased from -100 to $60^{\circ} \mathrm{C}$ at a heating rate of $5^{\circ} \mathrm{C} / \mathrm{min}$ under dry $\mathrm{N}_{2}$ gas.

\section{Results and Discussion}

Nitrogen adsorption-desorption isothermal measurement revealed that a BET surface area of neat-MPS used in this study is $933 \mathrm{~m}^{2} / \mathrm{g}$. This value is quite large compared with that of commercial particle-type silica (approximately $90 \mathrm{~m}^{2} / \mathrm{g}$ ) used in the previous study ${ }^{26)}$. In addition, the MPS had a distributed pore size of $6 \mathrm{~nm}$ and a total pore volume of $0.65 \mathrm{~cm}^{2} / \mathrm{g}$. From the results of elemental analysis, the elemental contents to the total weight of sample of organic compounds for neat-MPS and IL-MPS were detected as 1.1 and $2.7 \%$ on hydrogen (as $\left.\mathrm{H}_{2} \mathrm{O}\right), 2.3$ and $17.1 \%$ on carbon (as $\mathrm{CO}_{2}$ ), and 0 and $6.4 \%$ on nitrogen $\left(\right.$ as $\left.\mathrm{N}_{2}\right)$, respectively. The $\mathrm{EMIBF}_{4}$ content in total weight of IL-MPS was estimated to be $45.5 \%$. Small amounts of $\mathrm{H}$ and $\mathrm{C}$ in the neat-MPS are derived from small amount of residual surfactants or organic functional groups on silica surface such as hydroxyl or carbonyl. The nitrogen of IL-MPS was detected as $6.4 \mathrm{wt} \%$, and there was no nitrogen in the neat-MPS. Hence, a volume ratio of EMIBF4 (Density: $1.294 \mathrm{~g} / \mathrm{cm}^{3}$ ) in IL-MPS to the pore volume of MPS was calculated to be 1.11 .

SAXS diffraction patterns of the neat- and IL-MPS are shown in Figure 2-I. The spectra on neat-MPS powder show three peaks, indicated as (1 00$)$, (1 10$)$, and (2 000$)$ for a typical hexagonal structure. Both samples appear a strong peak at approximately 0.9 degree in the spectra. The periodic structure of the samples can be estimated from the one-dimensional correlation function $(\gamma)$, Figure 2-II ${ }^{27}$. This figure indicates that both fillers possess wellregulated and hexagonal crystal structure, because these shows very clear attenuation $\gamma$ curves. From the $\gamma$ curves, long period $(L)$, thickness of silica framework $(l)$, and pore diameter $(d)$ can be estimated. The data are summarized in

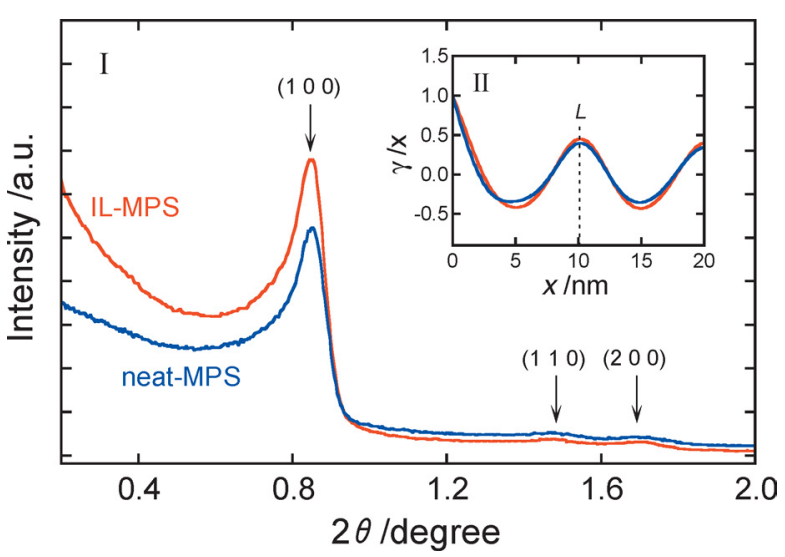

Figure 2. SAXS patterns (I) and one-dimensional correlation functions (II) of neat- and IL-MPS.

Table 1. Long period $(L)$, thickness of silica framework $(l)$, and pore diameter $(d)$ of neat- and IL-MPS estimated from the onedimensional correlation function of SAXS

\begin{tabular}{lccc}
\hline Sample & $L / \mathrm{nm}$ & $l / \mathrm{nm}$ & $d / \mathrm{nm}$ \\
\hline Neat-MPS & 10.2 & 2.6 & 7.6 \\
IL-MPS & 10.2 & 3.5 & 6.7 \\
\hline
\end{tabular}

Table 1 . Here, the $L(=l+d)$ was obtained from the $\mathrm{x}-$ coordinate of first peak top on the $\gamma$ curve. The two-phase structure, which consists of the high and low electron density phases whose one-dimensional length is represented as $l$ and $d$, can also be estimated from the distribution functions of electron density ${ }^{28)}$. Both fillers show the same $L$ of $10.2 \mathrm{~nm}$, and the value is good agreement with the value of approximately $10 \mathrm{~nm}$ which has previously been estimated from the TEM image ${ }^{24)}$. This indicates that the high-ordered framework in IL-MPS is maintained. However, there are apparent differences in $l$ and $d$ between neat- and IL-MPS. It is considered that the decrease in the $d$ of IL-MPS was probably caused by the insertion of $\mathrm{EMIBF}_{4}$ molecules into the nanopores. The insertion should decrease in the difference of electron density between the crystalline $\mathrm{SiO}_{2}$ walls and nanopores. In fact, it has been reported that the mesoporous inorganic fillers, which was inserted by some organic compounds, show the decrease in the $d$ as compared with the neat fillers $^{24)}$.

SEM photographs of the section for PEO composite films filled with neat- and IL-MPS are shown in Figure 3. The sectional image of neat-MPS composite clearly separates into black area as polymer matrix and white area as MPS (Figure 3a). Some MPS aggregators with size of from 10 to $20 \mu \mathrm{m}$ are also observed from this figure. Our previous report revealed that the neat-MPS consists of 

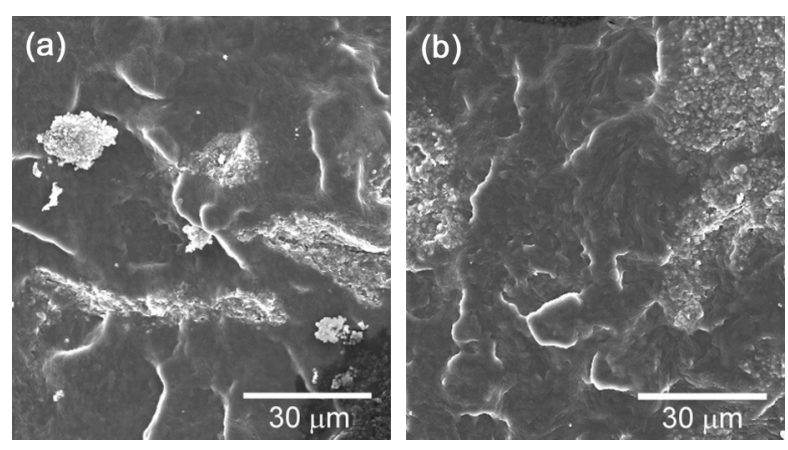

Figure 3. SEM photographs of $\mathrm{PEO}_{20} \mathrm{LiBF}_{4}$ composites filled with (a) $10 \mathrm{wt} \%$ neat-MPS and (b) $20 \mathrm{wt} \%$ IL-MPS (including $9.1 \mathrm{wt} \%$ $\mathrm{EMIBF}_{4}$ ).

many oval domains with relatively uniform sizes of from 1 to $2 \mu \mathrm{m}$, which are aggregated as network-like macrostructures ${ }^{24)}$. The neat-MPS seems to facilitate their aggregation into polymer, whereas it has a relatively large domain size compared with typical fillers such as carbon black or particle silica. On the other hand, Figure 3b indicates that the aggregators in IL-MPS composite are scarcely observed. The IL-MPS composite has relatively good dispersion state compared with the neat one, although it also shows two-phase separation. This should be caused by the modification with IL molecules, which probably act as surfactant for polymer-MPS interface.

Temperature dependence of ionic conductivity for the composites filled with neat- and IL-MPS are shown in Figure 4. The original sample, $\mathrm{PEO}_{20} \mathrm{LiBF}_{4}$, had a low conductivity, of the order of $10^{-7} \mathrm{~S} / \mathrm{cm}$ at room temperature, because of a transition at approximately $60^{\circ} \mathrm{C}$ corresponding to the melting temperature of crystalline PEO domains. Above the transition point, the sample shows relatively high conductivities and the temperature dependence essentially follows the VTF and/or WLF-type equations ${ }^{4,5)}$. In general, the conductivity is strongly related to the PEO chain mobility of the local structure, based on the ion-dipole interactions between cation and ether oxygen which have a considerable influence on ionic conduction ${ }^{4}$. Regarding neat-MPS-filled PEO composites, we have already reported that the MPS composites at $10 \mathrm{wt} \%$ contents show more than 30-fold higher conductivities than original $\mathrm{PEO}_{20} \mathrm{LiCF}_{3} \mathrm{SO}_{3}$ electrolyte at room temperature ${ }^{26)}$. It was concluded that extremely large surface area of MPS improves ionic conduction in PEO. Because, it has been reported by many researchers that the interface between polymer electrolyte phase and filler surface functions as ion (especially, cation) transport paths. At the interface, the filler surface probably acts as weak Lewis acid and interacts with the basic anions or OE

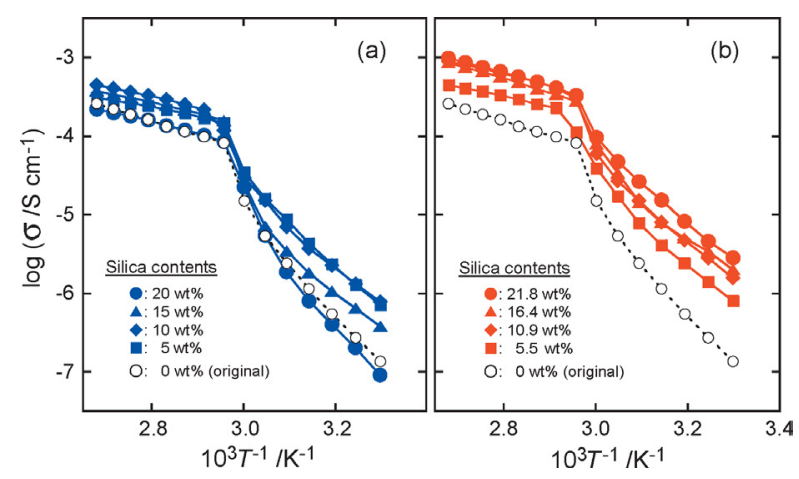

Figure 4. Temperature dependence of ionic conductivity $(\sigma)$ for $\mathrm{PEO}_{20} \mathrm{LiBF}_{4}$ composites filled with (a) neat-MPS and (b) IL-MPS.

chains. Therefore, cation transference number $\left(t_{+}\right)$should be enhanced because the cations can move faster through the interface ${ }^{13,14)}$. According to our previous report ${ }^{25)}$, it has been shown that the $t_{+}$of neat-MPS composite is certainly higher than that of the original PEO electrolyte. However, in this study, the composite filled with neat-MPS of $10 \mathrm{wt} \%$ show only 4 -fold higher conductivities than the original $\mathrm{PEO}_{20} \mathrm{LiBF}_{4}$, to be $7.9 \times 10^{-7} \mathrm{~S} / \mathrm{cm}$ at $30^{\circ} \mathrm{C}$ (Figure 4 a). Moreover, the composite filled with neat-MPS of 20 wt $\%$ shows slightly lower conductivities than the original. This reason is that the conductivity of original PEO-LiBF is approximately 10-fold higher than that of PEO$\mathrm{LiCF}_{3} \mathrm{SO}_{3}$ at room temperature, indicating that the MPS as filler scarcely affects on improvement in the ionic conduction. In the simple neat-MPS composites, unfortunately, the large surface area of MPS seems to be ineffectually used for the increase in the conductivity. On the other hand, the conductivities of IL-MPS-filled composites are higher than that of the original and neatMPS composites (Figure 4b). In particular, a composite filled with IL-MPS of $40 \mathrm{wt} \%$ (silica contents: $21.8 \mathrm{wt} \%$ ) shows the highest conductivities at entire measurement temperature. The large increases in the conductivity were probably caused by fast ion transport through IL-filled "nano-tunnels" within MPS. In addition, the slope of the Arrhenius plots, showing the apparent activation energy, decreases slightly with increasing IL-MPS contents.

Temperature dependence of storage modulus $E^{\prime}$ and loss modulus $E^{\prime \prime}$ for the composites filled with neat- and ILMPS are shown in Figure 5. Here, the $E^{\prime}$ is a measure of the recordable strain energy in a deformed sample film, and the $E^{\prime \prime}$ is related to the dissipation of energy as heat due to the deformation of material. In Figure 5a, both shoulders on $E^{\prime}$ curves and peaks on $E^{\prime \prime}$ curves at approximately $-25^{\circ} \mathrm{C}$ are due to the glass transition of amorphous PEO phase. Increases in $E^{\prime}$ and $E^{\prime \prime}$ values of 


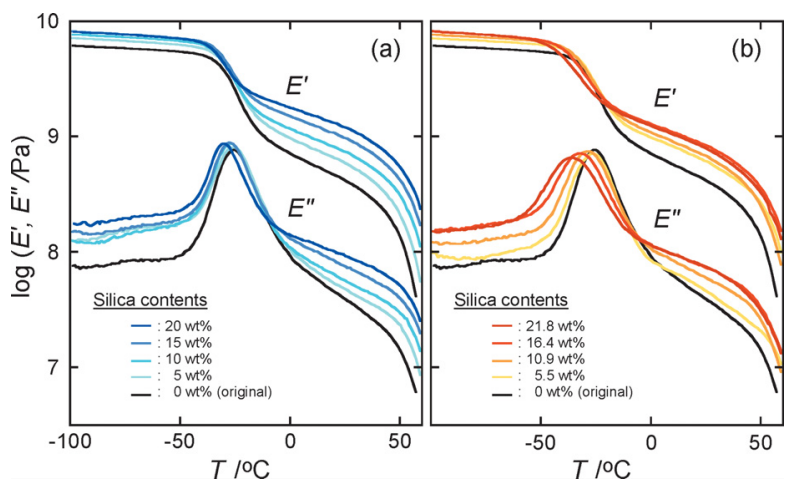

Figure 5. Temperature dependence of storage modulus $\left(E^{\prime}\right)$ and loss modulus $\left(E^{\prime \prime}\right)$ for $\mathrm{PEO}_{20} \mathrm{LiBF}_{4}$ composites filled with (a) neatMPS and (b) IL-MPS.

the composites are observed by the addition of neat-MPS at both glass and rubber regions. Because, the addition of filler gave rise to the reinforcement effect on polyether electrolytes as seen in the simple polymer composites. There are almost no changes in peak temperatures and maximum values of $E^{\prime \prime}$ curves for composites with different MPS contents. On the other hand, the reinforcement effect of IL-MPS on PEO composites was also shown and similar increases in both $E^{\prime}$ and $E^{\prime \prime}$ values were clearly confirmed (Figure $5 b$ ). This means that the added IL-MPS functions as filler for improvement in the storage modulus without leakage of IL from the nanopores. Peaks at the glass transition on $E^{\prime \prime}$ curves in Figure $5 \mathrm{~b}$ shift to lower temperature and become broad with increasing filler contents. This may be caused by the inside IL phase, which originally has low glass transition temperature (approximately $-70^{\circ} \mathrm{C}$ ).

Relation between silica (neat MPS) contents and conductivity or storage modulus for $\mathrm{PEO}_{20} \mathrm{LiBF}_{4}$ composites filled with neat- and IL-MPS are shown in Figure 6. The conductivity of original $\mathrm{PEO}_{20} \mathrm{LiBF}_{4}$ (silica contents: $0 \mathrm{wt} \%$ ) is low, to be approximately $10^{-7} \mathrm{~S} / \mathrm{cm}$ at $30^{\circ} \mathrm{C}$, because of its high crystallinity. Addition of neatand IL-MPS to the original electrolyte with only $5 \mathrm{wt} \%$ silica contents enhances the room temperature conductivities, since the filler addition gives rise to the inhibition of PEO crystallization. The neat-MPS composites show a maximum conductivity at approximately $10 \mathrm{wt} \%$ silica contents, which has been known in case of the PEO-based composites filled with nano-particles ${ }^{9}$. At higher silica contents above $10 \mathrm{wt} \%$, conductivities of neat-MPS composites decrease gradually with increasing contents, and are lower than that of the original $\mathrm{PEO}_{20} \mathrm{LiBF}_{4}$. This may be due to the aggregation of filler domains as insulator, which prevents the

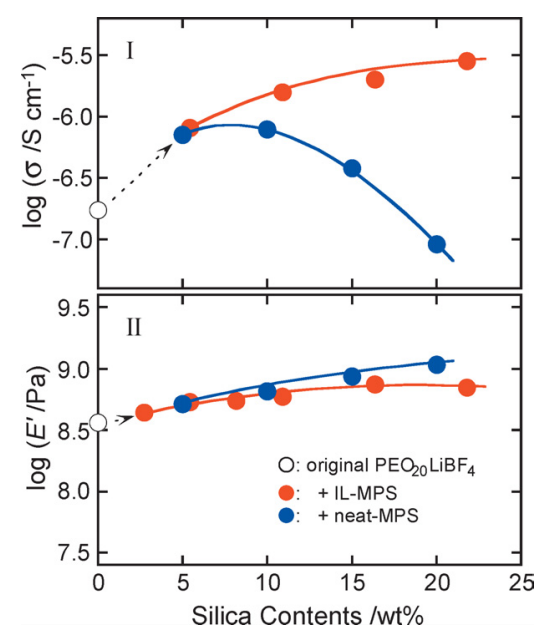

Figure 6. Relation between silica contents and $\log \sigma$ (I) or $\log E^{\prime}$ (II) of $\mathrm{PEO}_{20} \mathrm{LiBF}_{4}$ composites at $30^{\circ} \mathrm{C}$.

Table 2. Comparison of ionic conductivity $(\sigma)$ and storage modulus $\left(E^{\prime}\right)$ at $30^{\circ} \mathrm{C}$

\begin{tabular}{lcc}
\hline Sample & $\sigma / 10^{7} \mathrm{~S} \mathrm{~cm}^{-1}$ & $E^{\prime} / \mathrm{GPa}$ \\
\hline Original $\mathrm{PEO}_{20} \mathrm{LiBF}_{4}$ & 1.4 & 0.36 \\
+ IL-MPS, $10 \mathrm{wt} \% *$ & 8.1 & 0.54 \\
+ IL-MPS, $20 \mathrm{wt} \% *$ & 15.7 & 0.59 \\
\hdashline $\mathrm{PEO}_{20} \mathrm{LiBF}_{4}+\mathrm{EMIBF}_{4}(10 \mathrm{wt} \%)$ & 11 & 0.31 \\
\hline
\end{tabular}

* The added IL-MPS consists of EMIBF $_{4}$ of $45.5 \%$ and neat-MPS of $54.5 \%$.

segmental motion of local PEO chains and the ionic conduction in the amorphous phase. By contrast, the composites containing IL-MPS display a different conduction behavior. The conductivities are apparently improved by the addition of IL-MPS and increase with increasing silica contents. The improvement might be caused by fast migration of ions through inside IL phase or by ionic exchange between PEO matrix and the IL phase. The storage modulus $E^{\prime}$ of neat- and IL-MPS composites certainly increases with increasing silica contents at $30^{\circ} \mathrm{C}$. The addition of IL-MPS realizes the improvement in both conductivity and storage modulus in solid state, whereas the $E^{\prime}$ value of IL-MPS composites at higher silica contents is slightly lower than that of the neat one. The conductivity and $E^{\prime}$ values of original $\mathrm{PEO}_{20} \mathrm{LiBF}_{4}$ and the IL-MPS composites are summarized in Table 2. A simple mixture consisting of $\mathrm{PEO}_{20} \mathrm{LiBF}_{4}$ and pure $\mathrm{EMIBF}_{4}$ $(10 \mathrm{wt} \%)$ is also shown as reference data. The mixture apparently lowers the $E^{\prime}$ value, although the conductivity of the simple mixture is approximately 10 -fold higher than that of the original $\mathrm{PEO}_{20} \mathrm{LiBF}_{4}$. The mixture is obtained as a heterogeneous film showing liquid-polymer phase separation. On the other hand, the IL-MPS composites are 
obtained as homogeneous solid films and show at least 5fold increase in the conductivity and 1.5-fold increase in the $E^{\prime}$ value as compared with the original. Moreover, the addition of $20 \mathrm{wt} \%$ IL-MPS (Silica $: \mathrm{IL}=9.1: 10.9 \mathrm{wt} \%$ ) shows more than 10-fold in the conductivity and 1.6-fold in the $E^{\prime}$ value. This indicates that the addition made it possible to increase in the conductivity without loss of storage modulus.

We also revealed that the addition of IL-MPS should also be effective for rubbery materials. Epichlorohydrin rubber (Zeon, Gechron 3106) was used for a polymer matrix and was mixed with IL-MPS (2.8 wt $\%$, in this case) by using a mixing roll at $60^{\circ} \mathrm{C}$. DC conductivity of the ILMPS composite was approximately $10^{-6} \mathrm{~S} / \mathrm{cm}$ at room temperature. This value is more than 80 -fold higher than the neat Gechron $3106\left(1.2 \times 10^{-8} \mathrm{~S} / \mathrm{cm}\right)$. For practical use of the MPS composite, internal modification of the MPS using other organic compounds (instead of ionic liquid), improvement in dispersion state, and direction control of the dispersed MPS domains in the polymer matrix are needed.

\section{Conclusions}

We have used periodic mesoporous silica (MPS) and have prepared ionic liquid-modified MPS (IL-MPS) as novel fillers for improving ionic conductivity of PEO-based polymer electrolytes in solid state. The conductivity was markedly enhanced by addition of IL-MPS, to be more than $10^{-6} \mathrm{~S} / \mathrm{cm}$ at $30^{\circ} \mathrm{C}$ and at least $10 \mathrm{wt} \%$ silica contents, whereas the neat-MPS composites showed only 4-fold enhancement in the conductivity. The large enhancement may be due to the high-ordered channels within the MPS domains where fast migration of ions occurs through the IL phase. Dynamic mechanical measurements for neatand IL-MPS composites revealed that addition of fillers improves storage modulus of PEO-based electrolytes at room temperature. In particular, the addition of IL-MPS was able to realize the improvement in both ionic conductivity and storage modulus in the solid state.

\section{Acknowledgements}

One of the authors (Y. T.) acknowledges the financial support for young scientist from the Ministry of Education, Science, Sport, and Technology, Japan (No. 16750184). This study has been partially supported by The $21^{\text {st }}$ Century COE Program "Creation of Molecular Diversity and Development of Functionalities" at Tokyo Institute of Technology.

\section{References}

1) Wright, P. V.: Br. Polym. J., 7, 319 (1975).

2) Armand, M. B., Chabagno, J. M., Duclot, M. T.: "Fast Ion Transport in Solids", Eds. Vashishta, P., Mundy, J. N., Shennoy G. K., Elsevier, Amsterdam, (1979), p. 131.

3) Scrosati B., Schalkwijk, W.: "Advances in Lithium-Ion Batteries", Plenum Press, New York, (2002), p. 251.

4) Gray, F. M.: "Solid Polymer Electrolytes", VCH, New York, (1991), p. 95.

5) Bruce, P. G.: "Solid State Electrochemistry", Cambridge University Press, Cambridge, (1995), p. 95.

6) Takeoka, S., Ohno, H., Tsuchida, E., Polym. Adv. Tech., 4, 53 (1993).

7) Armand, M., Gorecki, W., Andreani, R.: "Proc. 2nd International Symposium on Polymer Electrolytes (ISPE-2)", Ed. Scrosati, B., Elsevier, London, (1990), p. 31.

8) Weston, J. E., Steele, B. C. H.: Solid State Ionics, 7, 75 (1982).

9) Capuano, F., Croce, F., Scrosati, B.: J. Electrochem. Soc., 138, 1918 (1991).

10) Borghini, M. C., Mastragostino, M., Passerini, S., Scrosati, B.: J. Electrochem. Soc., 142, 2118 (1995).

11) Croce, F., Appetecchi, G. B., Persi, L., Scrosati, B.: Nature, 394, 456 (1998).

12) Scrosati, B., Croce, F., Persi, L.: J. Electrochem. Soc., 147, 1718 (2000).

13) Croce, F., Persi, L., Scrosati, B., Serraino-Fiory, F., Plichta, E., Hendrickson, M. A.: Electrochim. Acta, 46, 2457 (2001).

14) Chung, S. H., Wang, Y., Persi, L., Croce, F., Greenbaum, S. G., Scrosati, B., Plichta, E.: J. Power Sources, 97-98, 644 (2001).

15) Bonneviot, L., Beland, F., Danumah, C., Giasson, S., Kaliaguine, S. Eds.: "Proc. 1st International Symposium on Mesoporous Molecular Sieves", Elsevier Science, Amsterdam, (1998), p. 1.

16) Ozin, G. A.: Adv. Mater., 4, 612 (1992).

17) Yanagisawa, T., Shimizu, T., Kuroda, K., Kato, C.: Bull. Chem. Soc. Jpn., 63, 988 (1990).

18) Kresge, C. T., Leonowicz, M. E., Roth, W. J., Vartuli, J. C., Beck, J. S.: Nature, 359, 710 (1992).

19) Zhao, D., Feng, J., Huo, Q., Melosh, N., Fredrickson, G. H., Chmelka, B. F., Stucky, G. D.: Science, 279, 548 (1998).

20) Zhou, Y., Antonietti, M.: Chem. Mater., 16, 544 (2004).

21) Asefa, T., MacLachlan, M. J., Coombs, N., Ozin, G. A.: Nature, 402, 867 (1999).

22) Miyata, H., Kuroda, K.: Chem. Mater., 12, 49 (2000).

23) Chu, P. P., Reddy, M. J., Kao, H. M.: Solid State Ionics, 156, 141 (2003).

24) Tominaga, Y., Asai, S., Sumita, M., Panero, S., Scrosati, B.: Electrochem. Solid-State Lett., 8, A22 (2005).

25) Tominaga, Y., Asai, S., Sumita, M., Panero, S., Scrosati, B.: J. Power Sources, in press.

26) Tominaga, Y., Igawa, S., Asai, S., Sumita, M.: Electrochim. Acta, in press.

27) Calleja, F. J. B., Vonk, C. G.: "X-ray Scattering of Synthetic Polymers", Elsevier, New York, (1989), p. 241.

28) Strobl, G.: "The Physics of Polymers", 2nd ed., Springer Verlag, Berlin, (1998), p. 408. 